\title{
DYNAMICS OF A' METAL OVERLAYER ON METALLIC SUBSTRATES - HIGH TEMPERATURE EFFECTS
}

TALAT S. RAHMAN, ${ }^{1,3}$ JOHN E. BLACI, ${ }^{2}$ AND ZENG JU TIAN ${ }^{2}$

${ }^{1}$ Brookhaven National Laboratory, Dept. of Physics, Bldg. 510A, Upton, NY 11973

${ }^{2}$ Brock University, Dept. of Physics, St. Catherines, Ontario L2S 3A1, Canada

${ }^{3}$ Permanent Address: Kansas State University, Dept. of Physics, Manhattan, KS 66506;

\section{ABSTRACT}

We have explored the structure and the dynamics of a bimetallic system consisting of a hexagonal (almost) overlayer of $A g$ on a square lattice $(N i(100)$ and $C u(100)$ ), as a function of the surface temperature. In each case the structure is "nearly" incommensurate giving rise to a low frequency Goldstone mode. Also, the overlayer atoms slosh back and forth over the substrate in a corrugated fashion. The calculated dispersion of the $\mathrm{Ag} /$ metal vertical mode, at room temperature, is in excellent agreement with experimental data. At higher temperatures floater atoms appear on top of the overlayer displaying a variety of cluster formations and also exchanges with the substrate atoms leading to surface disordering, interdiffusion and melting.

\section{INTRODUCTION}

An interesting question in surface alloy formation is how one type of metal atoms arrange themselves over the other because of the obvious differences in the lattice constants and the electronic structures. If these surface bimetallics lead to a commensurate or a nearly incommensurate structure then a related question is the stability of the interface as a function of surface temperature. Apparently, a commonly occuring combination is a hexagonal surface layer on a substrate with square symmetry. ${ }^{1}$ Our interest here is in two such systems, i.e. a monolayer of $A g$ on $N i(100)$ and on $C u(100)$. For $A g / N i$ the lattice mismatch is $16 \%$ while for $\mathrm{Ag} / \mathrm{Cu}$ it is $13 \%$. On forming a hexagonal arrangement on the (100) substrate, however, the overlayer becomes almost stress free. These structures have already been the subject of several experimental studies using low energy electron diffraction ${ }^{2,3}$ (LEED), electron energy loss spectroscopy ${ }^{3}$ (EELS) and scanning tunneling microscopy ${ }^{4}$ (STM), which point to the presence of a $c(2 \times 8)$ surface unit cell ${ }^{3,4}$ for $\mathrm{Ag}$ on $N i(100)$ and a $c(2 \times 10)$ structure ${ }^{2,3}$ for $A g$ on $C u(100)$, i.e. $A g$ atoms are row-aligned with the substrate atoms in the ratio of $7 \mathrm{Ag}$ to $8 \mathrm{Ni}$ and $9 \mathrm{Ag}$ to $10 \mathrm{Cu}$, respectively. Apart from structure, the dispersion of the $A g$ vertical mode along a high symmetry direction has also been measured. ${ }^{3}$

Our interest here is in the dynamics and the stability of these nearly incommensurate structures, particularly at higher temperatures. The earlier theoretical studies of these structures were based on pair potentials and the role of the rigid substrate was to provide surface corrugation. ${ }^{3,5,6}$ Our approach here is to use many-body potentials of the embedded atom (EAM) type for a molecular dynamics simulation in which all substrate and adsorbate atoms are dynamic. Starting with a row-aligned configuration at room temperature of the type predicted from data, we first calculate the structure factor and the dispersion of the $\mathrm{Ag} /$ metal vertical mode whose agreement with EELS data would test the reliability of the interatomic potential. Next we look for the Goldstone mode which is to be expected in incommensurate or nearly incommensurate structures. ${ }^{5}$ This is a mode localized in the surface layer and polarized along the incommensurate direction/plane whose frequency $\omega \Rightarrow 0$ as $\vec{Q}_{\| \prime} \Rightarrow 0\left(Q_{\|}\right.$is the wave vector polarized parallel to the surfa plane), for a totally incommensurate structure, and $\omega \Rightarrow$ finite value as $\vec{Q}_{\prime \prime} \rightarrow 0$, for a nearly incommensurate structure. We then analyze the mean square displacements of the atoms and the dispersion of the surface phonon along several high

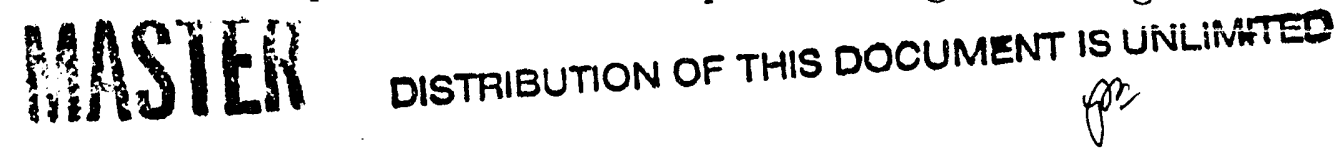


symmetry directions and compare these quantities made with those on the bare substrate. Finally, the simulations are extended to higher surface temperatures to observe anharmonic effects and to monitor the process of surface disordering.

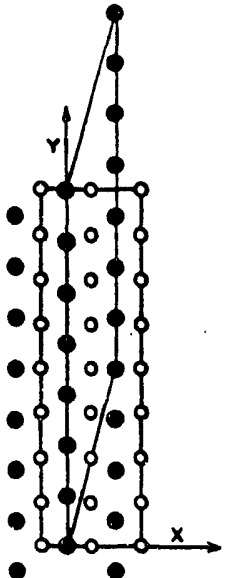

Fig. 12. The Lattice

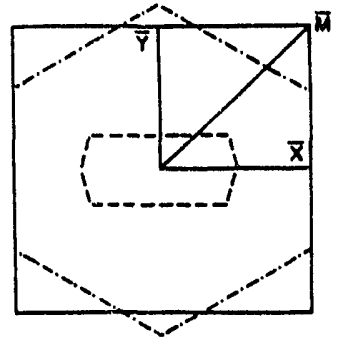

Fig. 1b. The Surface Brillouin Zope

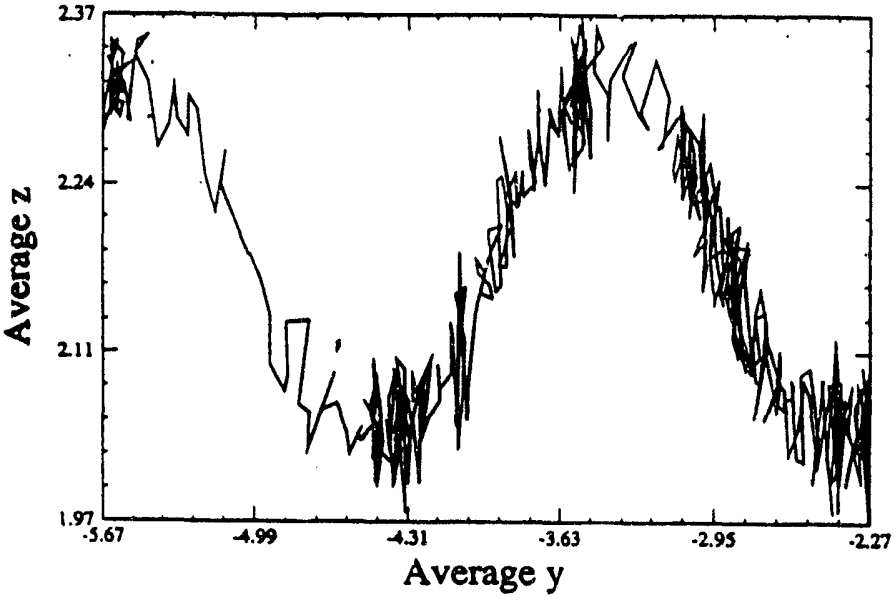

Fig. 2. Fluctuations in coordinates of 16 equivalent $\mathrm{Ag}$ atoms at $300 \mathrm{~K}$

In the next section we describe the model system and some details of the :alculations. Section III contains the results and is followed by some discussions in Section IV.

\section{MODEL SYSTEM AND CALCULATIONS DETAILS}

For clarity we will discuss mostly the $A g / N i(100)$ system and report on results for $\mathrm{Ag} / \mathrm{Cu}(100)$ only when appropriate. The surface unit cell shown in Fig. 1a consists of 7 $\mathrm{Ag}$ atoms arranged on top of $8 \mathrm{Ni}$ lattice spacing. Note that we have chosen [110] as the incommensurate direction and an equally favourable domain orientation would have the nearly incommensurate structure along [110]. In an experiment both types of domains exist and have to be accounted for in the analysis of the data. The Brillouin Zone for the hexagonal $A g$ layer together with that of the substrate is presented in Fig. $1 \mathrm{~b}$.

The molecular dynamics cell consists of $112 \mathrm{Ag}$ atoms arranged as in the Fig. 1a (4 units along the $x$-axis and 2 units along the $y$-axis) over 9 layers of $N i$ with 128 atoms/layer. Periodic boundary conditions are applied in the directions parallel to the surface. Classical equations of motion for all 1264 atoms are solved numerically using standard techniques. After the system has been equilibrated at the desired temperature, statistics are accumulated over longer runs of 50-200 ps at each temperature. The mean square vibrational amplitudes, the layer-by-layer structure factor and the surface phonon spectral densities are then calculated. The details of these calculations will be presented elsewhere. ${ }^{7,8}$

The interaction potential is obtained from the embedded atom method. ${ }^{9}$ The main virtue of this form for our purposes here is that it is many-body, albeit empirical, in nature and parameters do not have to be adjusted to reproduce surface phenomena.

\section{RESULTS}

We present here some of the results for $A g$ on $N i(100)$ at $300 \mathrm{~K}$ and at $1200 \mathrm{~K}-1700 \mathrm{~K}$. At lower temperatures the main emphasis is on the dynamics of the $c(2 \times 8)$ structure, which persists even at $1200 \mathrm{~K}$, while the high temperature studies examine the approach to surface disordering and melting. Note that the bulk melting temperature for $N i$ using EAM potentials is $1740 \mathrm{~K}$ (experimental value is $1726 \mathrm{~K}$ ).

\section{a. Sliding/Sloshing Motion and the Goldstone Mode}

An interesting conclusion from the analysis of the mean square displacements of the atoms is that at $300 \mathrm{~K}$ the $A g$ overlayer slides back and forth over the $N i$ substrate along the nearly incommensurate direction. The center of mass of the $N i$ layers move in the 
opposite direction to keep the net center of mass of the system stationary. Also, as each $A g$ atom moves a distance of about $3.4 \AA$ from a bridge site to a hollow site it does so in a corrugated manner as shown in Fig. 2. It lies low above $N i(100)$ at the hollow site and rides higher at the bridge position.

The calculated phonon spectral densities near the zone center $\left(\vec{Q}_{\prime \prime} \rightarrow 0\right)$ exhibits a very low frequency mode $\left(1.5 \mathrm{~cm}^{-1}\right.$ at $\left.300 \mathrm{~K}\right)$ which is polarized along the $y$-axis (the nearly incommensurate direction). This is the Goldstone mode for $A g$ on $N i(100)$. Its finite frequency corresponds to the lock-in energy for the translation of the overlayer along the nearly incommensurate direction. For $A g$ on $C u(100)$ the frequency of this : node is $1.3 \mathrm{~cm}^{-1}$ at $300 \mathrm{~K}$.
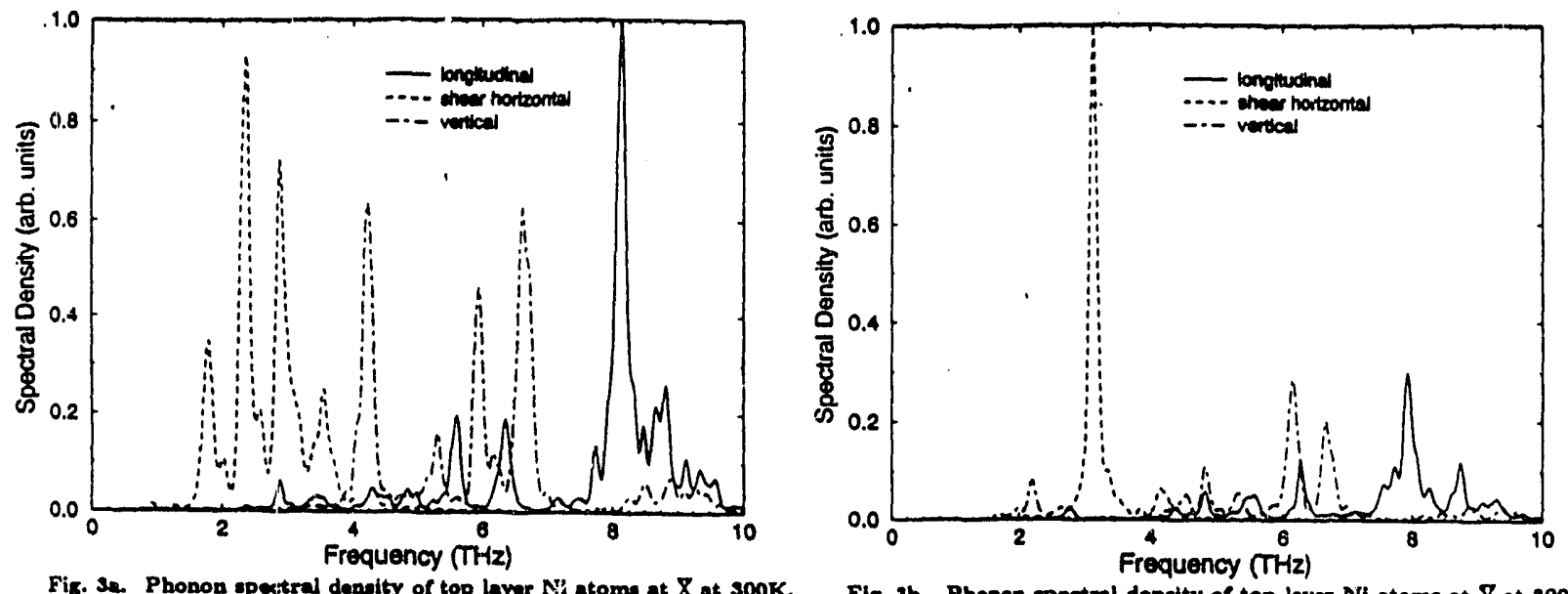

Fig. 3b. Phonon opectral dennity of top layer Ni atome at $\mathrm{Y}$ at $300 \mathrm{~K}$.

\section{b. Surface Phonon Dispersion}

We have calculated the dispersion of the surface phonons at $300 \mathrm{~K}$ and $1200 \mathrm{~K}$ along three high symmetry airections in the substrate Brillouin Zone: $\bar{\Gamma}-\bar{M}, \bar{\Gamma}-\bar{X}$, and $\bar{\Gamma}-\bar{Y}$. While for the bare subrcrate $\bar{X}$ and $\bar{Y}$ are equivalent, with the hexagonal overlayer they ar not and $\bar{Y}$ is no longer at the overlayer zone boundary (see Fig. 1b). Also, the $\bar{\Gamma}-\bar{M}$ direction is significant because it is equivalent for the two domain orientations, thereby facilitating experimental measurements of the surface modes along this particular direction. Along this direction our calculated dispersion of the $\mathrm{Ag} / \mathrm{Ni}$ and the $\mathrm{Ag} / \mathrm{Cu}$ vertical mode $\mathbf{e}^{8,10}$ is in excellent agreement with the EELS data. ${ }^{3}$

In Fig. 3, we present the spectral densities for the $N i$ atoms, in the layer directly below the $A g$ overlayer, at $\bar{X}$ and $\bar{Y}$ points in the two-dimensional Brillouin Zone. In each figure the "longitudinal" and the "shear horizontal" modes lie in the surface plane, polarized, respectively, parallel to the wave-vector and normal to it. The "vertical" mode is polarized along the surface normal. At $\bar{X}$ the shear horizontal mode is softer than the corresponding one at $\bar{Y}$, and it is also split into several modes. This is a result of the nearly incommensurate $(1 \times 8)$ arrangement along the $y$-axis along which the shear horizontal mode at $\bar{X}$ is polarized. The shear horizontal mode at $\bar{Y}$ is polarized along the $x$-axis which is the commensurate direction for the $A g$ overlayer. Thus, there is no splitting in this mode or a softening in the frequency. At $\bar{Y}$, the shear horizontal mode resembles more the same feature ${ }^{11}$ on bare $N i(100)$. The Rayleigh wave at $3.9 \mathrm{THz}$ on the bare surface, appears prominently at a slightly higher frequency at $\bar{X}$ (Fig. 3a) and not at $\bar{Y}$ (Fig. 3b). Additionally, two other vertical modes are present at $\sim 6 \mathrm{THz}$ and $6.8 \mathrm{THz}$ at both $\bar{X}$ and $\bar{Y}$ for the bimetallic system.

The richness in the phonon spectral densities at $\bar{X}$ and $\bar{Y}$ call for experimental measurements to validate the claims made here. However, a word of caution is that the presence of the other domain orientation would cause considerable mixing in the signals from the two inequivalent symmetry points. 
The spectral densities for the $l g$ atoms at $\bar{X}$ and $\bar{Y}$ and for both $A g$ and $N i$ atoms at $\bar{M}$ further reveal the nature of the $\ldots$. ding between $A g$ and $N i$ atoms and the pronounced directional asymmetry. At $1200 \mathrm{~K}$ all modes broaden but not all to the same extent. These details can be found in Ref. [7].

\begin{tabular}{|c|c|c|cc|}
\hline \multicolumn{5}{|c|}{ Mean Square Displacement $(\mathrm{Ag})$} \\
(In Units of $\left.10^{-2} \mathrm{~A}^{2}\right)$ \\
\hline $\mathrm{T}$ & $\left\langle u_{x}^{2}\right\rangle$ & $\left\langle u_{y}^{2}\right\rangle$ & $\left\langle u_{s}^{2}\right\rangle$ \\
\hline $3 \mathrm{~K}$ & .004 & .011 & .014 & \\
\hline $300 \mathrm{~K}$ & .830 & 1.600 & 2.300 & $(1.3)$ \\
\hline $1200 \mathrm{~K}$ & 5.500 & 10.500 & 8.300 & \\
\hline $\mathrm{Ag}(111) 300 \mathrm{~K}$ & 1.096 & 1.173 & 1.590 \\
\hline
\end{tabular}

\section{c. Mean Square Vibrational Amplitudes}

The variation of the mean square vibrational amplitude of atoms with temperature is a measure of the anharmonicity of the interaction potential. For harmonic systems this scaling is linear. The numbers in Table I illustrate that even at $300 \mathrm{~K}$ the $A g$ vibrations are anharmonic and the anharmonicity is largest along $x$ - the commensurate direction. More significant is the enhanced vibrational amplitude at all temperatures along the $y$ axis resulting from the nearly incommensurate arrangement. Note that the $y$ motion of the center of mass of the $A g$ luyer has been already subtracted. The value $1.3 \times 10^{-2}$ for $\left\langle u_{z}^{2}\right\rangle$ results when the slow vertical oscillation between bridge and hollow sites is subtracted out. Comparison of the mean square vibrational amplitude of the $A g$ atoms on $N i(100)$ with those of the top layer $A g$ atoms on $A g(111)$ shows that $\left\langle u_{x}^{2}\right\rangle$ for the former is in fact smaller than that of the latter. The vibrational amplitudes of the $N i$ atoms in the layer directly below the $A g$ are also anharmonic and anisotropic. ${ }^{7}$

\section{d. Surface Disordering}

We now turn to the atomic trajectories in the high temperature range, i.e. $1200 \mathrm{~K}$ to $1700 \mathrm{~K}$. At $1200 \mathrm{~K}$ the first 50 ps run after equilibration was rather quiet as only one $\mathrm{Ag}$ atom left the overlayer to float over it for about $26 \mathrm{ps}$ and then to settle back into the layer. Subsequent runs for a total of 150 ps exhibited much more activity with several floater atoms windering, dimerizing, clustering, unclustering, and so forth. There were also exchange processes in which $\mathrm{Ni}$ atoms would move into the overlayer and $\mathrm{Ag}$ atoms would move in in Fig. 4. Note that at this temperature the structure is on the average $c(2 \times 8)$ although large distortions are present.

At $1300 \mathrm{~K}$, the disordering of the surface was more pronounced than at $1200 \mathrm{~K}$. Occasionally a silver atom would be trapped even in the second layer of the $N i$ substrate and several $\mathrm{Ni}$ atoms found their way into the overlayer. There were also clustering of $\mathrm{Ni}$ and of $\mathrm{Ag}$ atoms. As the temperature was raised to $1500 \mathrm{~K}$, all these processes became more frequent making the surface pretty disordered. At $1300 \mathrm{~K}$, and even more so at $1500 \mathrm{~K}$, there were a large number of $N i$ atoms in the overlayer and also floating above the overlayer. Similarly some $A g$ atoms penetrated into the third substrate layers while most floated above in clusters. Despite the increased activity in the surface layers, the layers below second $N i$ layer were relatively calm even at $1500 \mathrm{~K}$. The situation changed completely at $1700 \mathrm{~K}$, which is close to the bulk melting temp rature of $N i . A g$ and $\mathrm{Ni}$ atoms were intermingled everywhere, the surface was completely disordered (melted) 
and there was a large outward vertical expansion of the system. These characteristics are presented below in Fig(s). 5-8, which show a side view of the system of $9 \mathrm{Ni}$ layers and 1 $A g$ overlayer. Here we have two free surfaces but the $A g$ overlayer has been placed at the top surface only. The lighter atoms are $\mathrm{Ni}$. Note the dramatic change in the ordering at $1700 \mathrm{~K}$ at both the lower and the upper surface. In Fig. 8 the lower most atom is an $A g$ atom that has gone down 9 layers. In Fig. 9 the average linear density of the $A g$ atoms as a function of the height above the $N i$ substrate is given. The averaging was done over $50 \mathrm{ps}$. At $1300 \mathrm{~K}$, there is only a trace of $\mathrm{Ag}$ in the first substrate layer and some have become "floaters" with an average height of $5 \AA$, while most $A g$ most are at $\sim 3 \AA$ from the starting configuration of the top layer substrate atoms. At $1500 \mathrm{~K}$, there is still a concentration of these atoms around $3.5 \AA$, but at $1700 \mathrm{~K}$ the curve looses its peaks at $A g$ atoms diffuse everywhere.

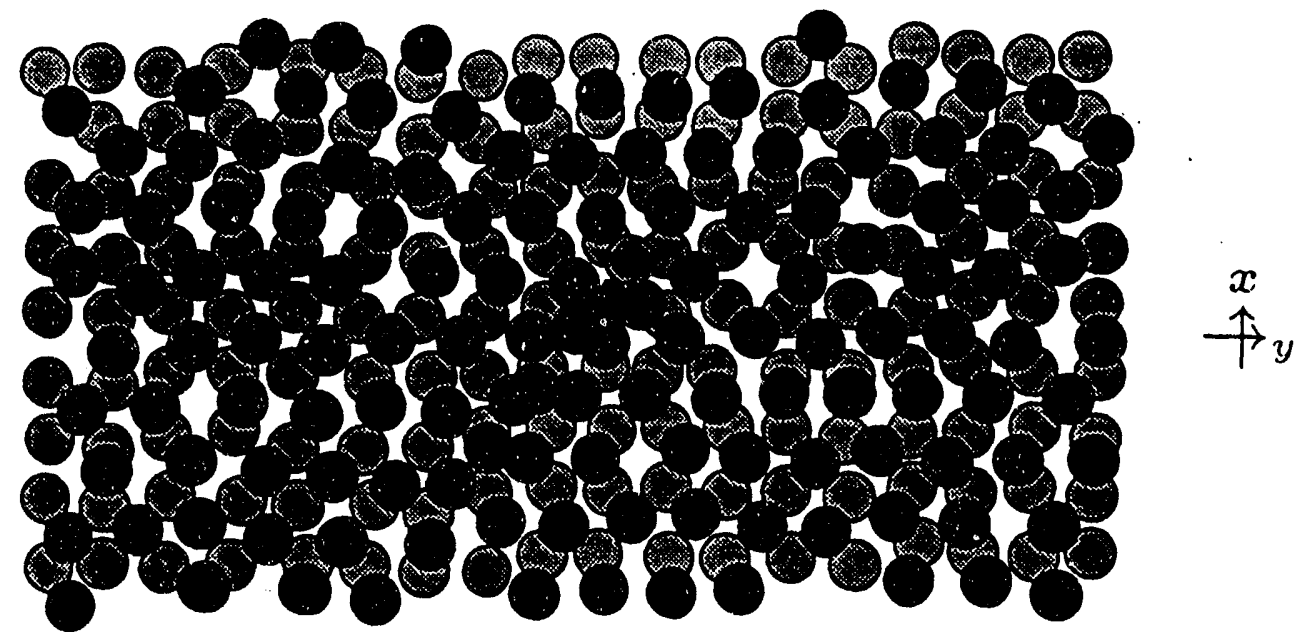

Fig. 4. Top view of $\mathrm{Ag} / \mathrm{Ni}(100)$ at $1200 \mathrm{~K}$.

\section{DISCUSSION}

We have found the $A g / N i(100)$ system very dynamic at all temperatures here. At $300 \mathrm{~K}$, the sliding/sloshing of the overlayer on the substrate along the incommensurate axis, the presence of the Goldstone mode and the richness in the surface phonon spectral densities offer several opportunities to understand the characteristics of such bimetallic systems both experimentally and theoretically. At higher temperatures, the approach to surface disorder indicates the interdiffusion of $A g$ and $N i$ atoms with the rate increasing with temperature. Since in our simulations one free surface behaves like clean $N i(100)$, Fig(s).6-8 also contrast the relative calm of that surface compared to the other, except at $1700 \mathrm{~K}$ when both surfaces are disordered.

It will be interesting to follow these atomic trajectories over much longer times and also study the effects of the finite size of the system, but such work will be meaningful when coupled to similar experimental studies.

\section{ACKNOWLEDGEMENT}

This work was supported in part by the Division of Materials Sciences, U. S. Department of Energy, under Contact No. DE-AC02-76CH00016, the National Science Foundation under Grant No. DMR9120440, and the Natural Sciences and Engineering Research Council of Canada. Computer time was provided by the Pittsburgh Supercomputing Center. We would like to thank Stephen Foiles and Murray Daw for supplying us with the EAM codes and also a graphic software, and Michael Weinert for helpful discussions. 


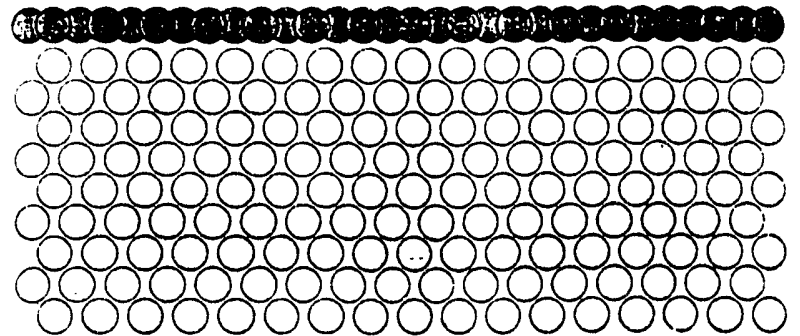

Fig. 5. Initial configuration of $\mathrm{Ag} / \mathrm{Ni}(100)$ at $1700 \mathrm{~K}$

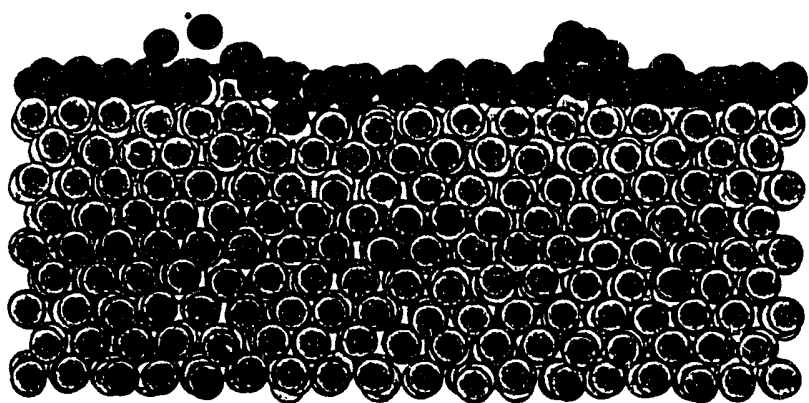

Fig. 6. Side view of $\mathrm{Ag} / \mathrm{Ni}(100)$ at $1300 \mathrm{~K}$ after 50 ps.

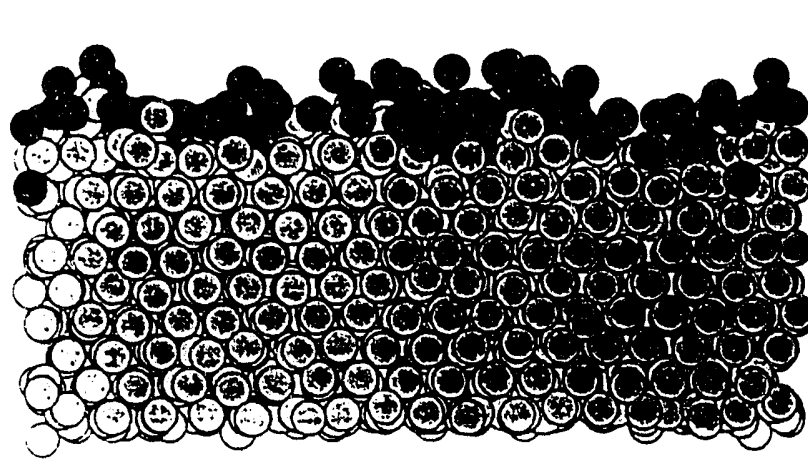

Fig. $\mathrm{i}$. Side view of $\mathrm{Ag} / \mathrm{Ni}(100)$ at $1500 \mathrm{~K}$ after 50 ps.

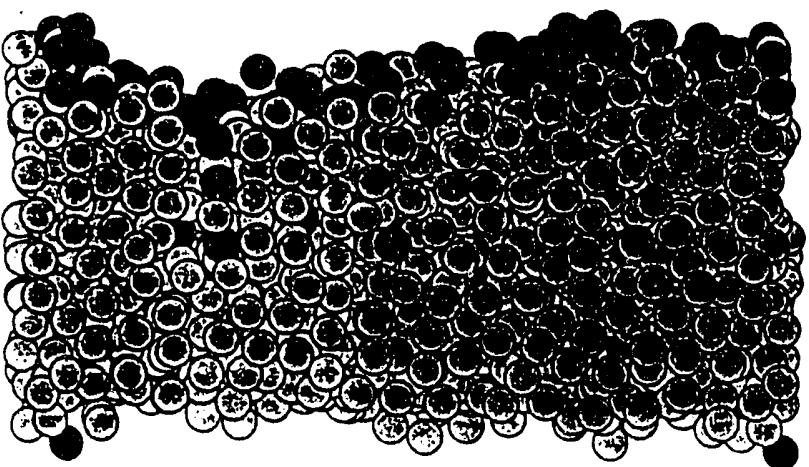

Fig. 8. Side view of $\mathrm{Ag} / \mathrm{Ni}(100)$ at $1700 \mathrm{~K}$ after $50 \mathrm{ps}$.

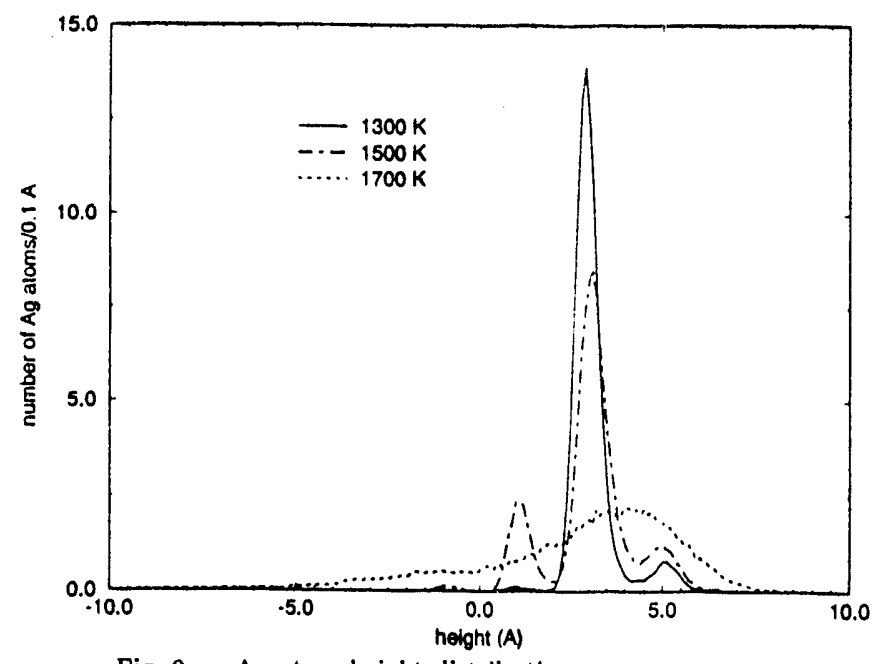

\section{REFERENCES}

Fig. 9. Ag atom height distribution.

1. H. Ohtani, C.-T. Kao, M. A. van Hove, and G. A. Somorjai, Prog. Sur. Sci. $2 \underline{23}, 155$ (1986).

2. P. W. Palmberg and T. N. Rhodin, J. Chem. Phys. $\underline{49}, 134$ (1968).

3. W. Daum, J. Electron Spectrosc. Related Phenomena 44 271 (1987); J. E. Black, D. L. Mills, W. Daum, C. Stuhlmann, and H. Ibach, Sur. Sci. 217, 529 (1989).

4. A. Brodde, G. Wilhelmi, D. Badt, H. Wengelnik, and H. Neddermeyer, J. Vac. Sci. Tech. B $\underline{9}, 920$ (1991).

5. J. E. Black and D. L. Mills, Phys. Rev. B $\underline{2}$, 5610 (1990).

6. J. E. Black, A. Janzen, and P. Bopp, Sur. Sci. 259, 371 (1992).

7. T. S. Rahman and J. E. Black, (to be published).

8. T. S. Rahman, J. E. Black, and Z. Tian, (to be published).

9. M. S. Daw and M. I. Baskes, Phys. Rev. B29, 6443 (1984); S. M. Foiles, M. I. Baskes, and M. S. Daw, Phys. Rev. B $\underline{33}, 7983$ (1986).

10. L. Yang, T. S. Rahman, and J. E. Black, Sur. Sci. (to appear).

11. Z. Tian and T. S. Rahman, (to be published). 


\section{DISCLAIMER}

This report was prepared as an account of work sponsored by an agency of the United States Government. Neither the United States Government nor any agency thereof, nor any of their employees, makes any warranty, express or implied, or assumes any legal liability or responsibility for the accuracy, completeness, or usefulness of any information, apparatus, product, or process disclosed, or represents that its use would not infringe privately owned rights. Reference herein to any specific commercial product, process, or service by trade name. trademark, manufacturer, or otherwise does not necessarily constitute or imply its endorsement, recommendation, or favoring by the United States Government or any agency thereof. The views and opinions of authors expressed herein do not necessarily state or reflect those of the United States Government or any agency thereof. 

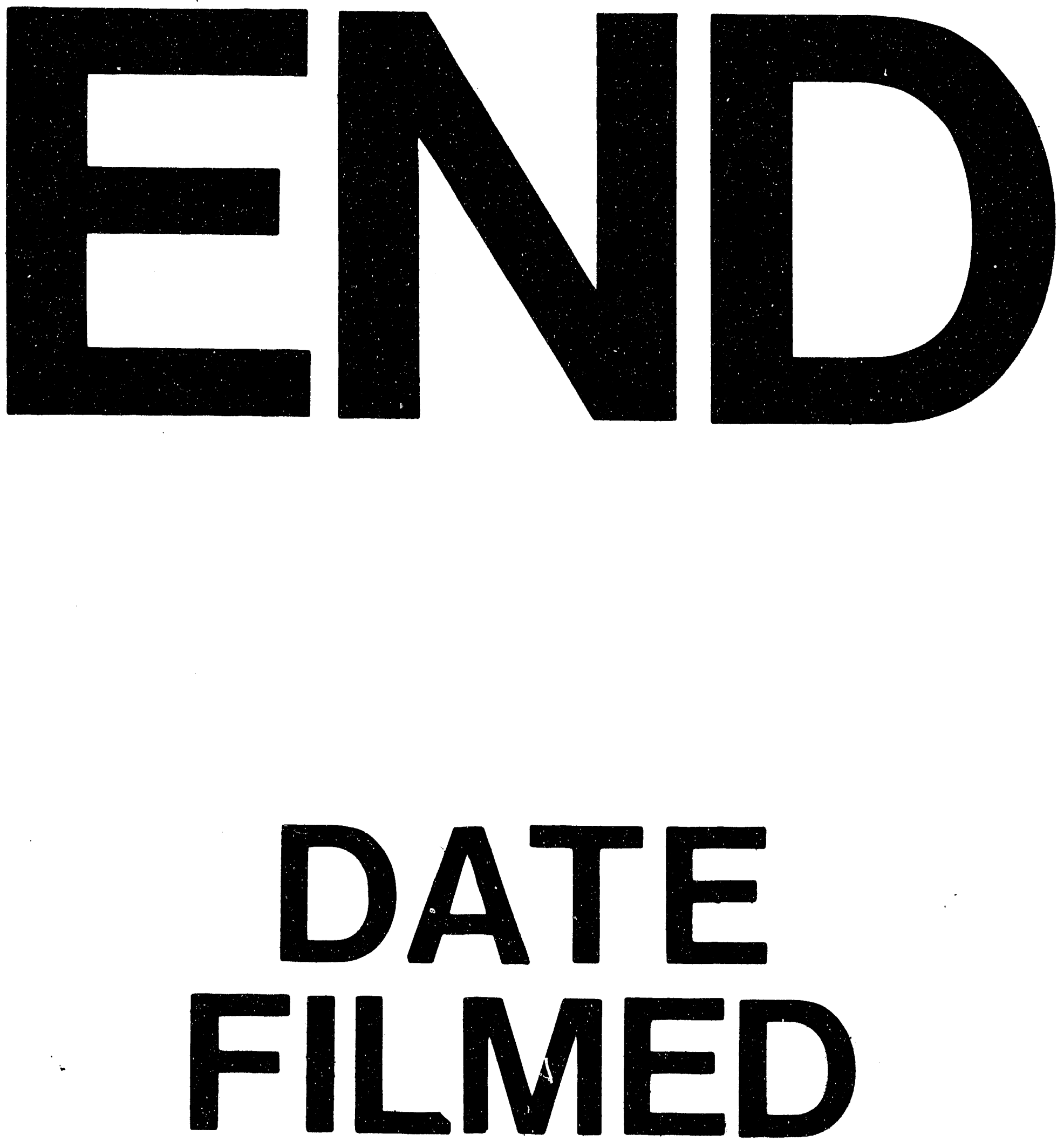

1

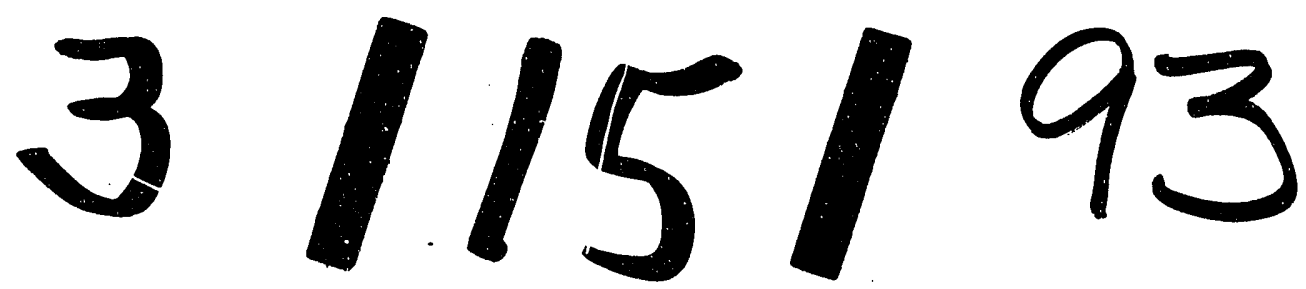


